\title{
A Hybrid Simulation of Converter-Interfaced Generation as the Part of a Large-Scale Power System Model
}

\author{
Igor Razzhivin*, Alisher Askarov, Vladimir Rudnik, Aleksey Suvorov \\ School of Energy and Power Engineering, National Research Tomsk Polytechnic University, Tomsk, Russian Federation \\ Received 10 March 2021; received in revised form 14 September 2021; accepted 15 September 2021 \\ DOI: https://doi.org/10.46604/ijeti.2021.7276
}

\begin{abstract}
This study aims to propose an alternative hybrid approach to model renewable energy sources (RESs), which provide the most reliable results in comparison with the existing simulating tools. Within the framework of this approach, a specialized hybrid processor for modeling converter-interfaced generation (CIG) is developed. This study describes its structure and validation in the test system by comparing the results with commercial modeling tools, and also presents experimental studies of its operation as parts of the practical power system. The results obtained confirm the adequacy of the developed tools.
\end{abstract}

Keywords: power system, converter-interfaced generation, time-domain simulation, hybrid approach

\section{Introduction}

The annually growing level of energy consumption leads to the need to introduce additional generation. At the same time, one of the main directions is the rapid increase in the penetration of power generating units based on renewable energy sources (RESs). The dominant installations are those that use wind and solar energy [1], most of which do not have a direct connection to grids (e.g., type 4 wind turbine generators (WTGs) and photovoltaic (PV) units [2]) and use power converters, mainly voltage source converters (VSCs), for connection. This feature causes a significantly different dynamics of the operation of such facilities in general compared to the conventional part of the electric power system (EPS), which becomes one of the main causes of challenges in operating state regulation and emergency control of modern EPSs [3]. Therefore, the assessment of the converter-interfaced generation (CIG) impact on power systems requires separate study and attention.

In connection with the above, it becomes necessary to carry out a comprehensive analysis and research directly related to the assessment of the impact of CIG penetration in modern power systems in a wide variety of operating conditions and disturbances. In this case, the reliable information about all states and processes in power systems is needed, and the only possible way to obtain the information is mathematical modeling. Thus, the vast majority of research is carried out using various specialized commercial digital software programs (DSPs) and hardware-software tools (HSTs) for modeling states and processes on the example of EPS mathematical models of various scales and levels of detail. At the same time, the questions of the reliability of the obtained modeling results remain open in connection with the simplifications and limitations used in various DSPs and HSTs. Also, one of the challenges is the questions about the sufficient level of detail of the models of power system components and the scale of the aggregate EPS as a whole, which would make it possible to comprehensively assess the efficiency and reliability of the CIG operation as parts of a practical EPS.

* Corresponding author. E-mail address: lionrash@tpu.ru

Tel.: +7-3822-701777(3438) 
The main purpose of this study is to develop and further use the hybrid simulation tools to model the analog, digital, and physical levels, in order to obtain the most reliable and complete information about the states and processes in power systems with CIGs. Such tools will be based on the simulation of a single range of processes without decomposition, ranging from electromagnetic to electromechanical transients. The simulation is associated with the reasons described in more detail below. The study also takes account of the specificity of operation of all associated CIG equipment in different operating states and disturbances in order to understand the interaction between CIG and the rest of power system.

This study is organized as follows. The existing simulation approaches of CIGs and power systems are presented in Section 2. Section 3 describes the concept of hybrid approach for a power system simulation. A specialized processor (SP) providing a simulation of a type 4 WTG created according to the hybrid approach is shown in Section 4 . Section 5 presents the validation results of the developed SP by the comparison with a credible simulation tool widely used for EPS modeling real-time digital simulator (RTDS), and presents the experimental studies of the developed hybrid tools as the part of a large-scale practical power system. Section 6 concludes the study.

\section{State-of-the-art of CIG Simulation}

Currently, the study of EPSs with CIGs is carried out mainly with the help of digital modeling tools: (1) DSPs of positive-sequence power system simulation; (2) DSPs and HSTs of electromagnetic transient power system simulation. Such digital DSPs and HSTs are based on the application of various numerical methods for solving the systems of nonlinear algebraic-differential equations that form a mathematical model of each element and EPS as a whole and describe the range of processes occurring in them. However, despite the high level of the development of these tools, they are characterized by certain simplifications and limitations during the process of solving the aggregate EPS mathematical model, which can significantly affect the completeness and reliability of the information obtained with the help of them. Moreover, in the context of the development of modern EPSs, the use of power converters in the structure of various RESs (type 3 and type 4 WTGs, PV units, energy storage systems, etc.) causes additional difficulties in the field of power system modeling, which can negatively affect the obtained results. On the one hand, they are associated with the need to simulate a large-scale aggregate EPS model, which takes account of the complex topology of the network and number of facilities located; on the other hand, with the necessary and sufficient level of detail of mathematical models of the simulated elements and EPS in general.

The effective means of power system mathematical modeling in the first direction are DSPs of positive-sequence power system simulation, which are widely used in world practice. These DSPs allow simulating practical large-scale EPSs, taking account of various mathematical models of conventional facilities, as well as the necessary control systems of different configurations and structures. The studies carried out using such DSPs in the conventional power systems were mainly focused on the time scale of the electromechanical transients - from milliseconds to several minutes [4]. The latter is due to the predominant impact of synchronous generators and their control systems on the dynamic properties of such EPSs. This makes it possible to simplify the EPS model and speed up the modeling process itself. The key assumption is that only the fundamental frequency component $(50 \mathrm{~Hz}$ or $60 \mathrm{~Hz})$ dominates the electrical network. It follows from that the network itself can be significantly simplified, and all variables are represented as steady-state phasors. With this approach, high-frequency switching and electromagnetic transients are not reproduced. In addition, the use of SPs of positive-sequence power system simulation is associated with a number of simplifications and limitations, even for the elements of the conventional part of EPS. The main characteristics of SPs are as follows [5]:

(1) Neglecting the transformation electromotive force in the equations of the stator circuit of electrical machines.

(2) The inability of determining a multi-mass shaft model of electrical machines. 
(3) Taking account of only the magnetic saturation in electrical machines caused by the main magnetic flux (often only in the d-axis), and neglecting the saturation caused by the leakage magnetic flux.

(4) Using single-line models instead of three-phase models, and accordingly using the method of symmetrical components.

(5) Simulating the network elements in forms of static models and corresponding algebraic equations instead of the differential ones.

Despite this, the results obtained for conventional EPSs were acceptable as a rule since high-frequency electromagnetic and electromechanical transients were not interrelated. However, in some cases, a significant error is still possible, mainly due to the impact of the network component, the elements of which are simulated in the form of static models [6].

To consider modern EPSs, by taking account of the relatively new RES-based generation facilities, generic models of these elements have been developed and are currently being modified for DSPs of positive-sequence power system simulation [7]. Such models can take account of the specifics of CIG operation in the context of the properties and capabilities of positive-sequence modeling. At the same time, these models have significant simplifications and limitations since they are reproduced in the form of controlled current or voltage source, which does not have physical analogues and mimics the CIG operation in the point of connection to the network. Such a simplification leads to the exclusion of the simulation of the electromagnetic stage of processes for these facilities, which include the dynamics of the power converter operation and their fast-response control systems, as well as the dynamics of a DC-link. The range of the simulated processes to which the generic models respond is very limited (from 0.1 to $3 \mathrm{~Hz}$ [7]), and significantly affects the completeness and reliability of the information. The latter is due to the fact that the characteristic time scale of operation of new CIG becomes much smaller than that of the conventional synchronous generation, and has already been from a few dozen to hundreds of microseconds. As a result, the dynamic of operation has already covered high-frequency switching and electromagnetic transients, which are always ignored in the SPs of positive-sequence power system simulation. Thus, the range of tasks that can be solved with the help of positive-sequence modeling for modern EPS with CIG is very limited.

At the same time, it is noted in the work of Xiong et al. [8] that taking account of the electromagnetic stage of processes for CIG is an important aspect due to the changes in the dynamic properties of modern EPSs, as well as the imposition of time scales of electromagnetic and electromechanical transients. The latter naturally leads to the need to consider a single range of processes without its decomposition into wave, electromagnetic, electromechanical, and thermodynamic phenomena. Thus, the use of DSPs of electromagnetic transient power system simulation in the framework of the second direction makes it possible to simulate the detailed models of both the conventional part of the EPS and CIG without any significant simplifications. However, for the simulation of a detailed aggregate EPS mathematical model, e.g., even of the regional scale, using DSPs of electromagnetic transient power system simulation is time-consuming and inevitably associated with significant computational difficulties [9]. There is also always the problem of the presence of a truncation error of the numerical integration methods and its accumulation during the simulation of long-term processes [10]. Taken together, this leads to the need for reducing the scale of the considered EPS, simplifying the regulation and control systems, neglecting the protection systems, using the only static loads, limiting the modeling intervals, etc. Thus, the mathematical modeling of EPS using DSPs of electromagnetic transient power system simulation refers to the consideration of small equivalent systems. At the same time, it has been theoretically and experimentally proved that the processes in a practical large-scale EPS and its simplified equivalent differ significantly [11].

The use of multiprocessor HSTs of electromagnetic transient power system simulation makes it possible to avoid some of the identified shortcomings inherent in the DSPs, especially in the context of computing the power and calculation speed [12]. These HSTs carry out the EPS modeling in real time, and provide the opportunity both to simulate the detailed models of the EPS elements with the necessary regulation and control systems and to consider the large-scale EPS models. At the same time, the scale of the studied EPS is limited by the hardware capabilities of the HSTs, which can be expanded. In this case, the main issue is the cost of such simulators. Moreover, the following challenges remain relevant when using HSTs: 
(1) Implementing the high-speed synchronous data exchange between the computing modules in real time.

(2) Synchronizing the calculation and data exchange between the individual parts of the EPS solved with different integration steps. For adequate simulation of facilities with power converters, a small integration step is required; therefore, usually in the HSTs these elements are solved with a small step (from $10 \mu \mathrm{s}$ and less), and the rest of the studied EPS are solved with a large step (from $50 \mu$ s and more).

(3) Converging the solution of the aggregate EPS model without the use of significant simplifications.

(4) The presence of a truncation error of the numerical integration methods used.

The indicated simplifications and limitations can lead to the errors in the results of modeling states and processes in the modern EPSs to one degree or another. At the same time, it should be noted that, depending on the specific tasks to be solved, the use of appropriate tools for positive-sequence modeling or electromagnetic transient modeling make it possible to achieve the results as close as possible to the real data due to the appropriate configuration of the studied EPS model during its validation process [13].

For the most reliable modeling of states and processes in modern EPSs, by taking account of the detailed models of elements as parts of the large-scale aggregate EPS model, new software and hardware tools are being developed to expand the capabilities of the existing DSPs and HSTs. For example, Yang et al. [14] divided a single EPS mathematical model into the subsystems calculated by different numerical integration methods and with different integration steps, within the framework of a single HST using field-programmable gate array. Sun et al. considered [15] a similar approach, but conducted it with the use of graphic processing units since the units allow achieving a higher computational speed with a smaller integration step. In addition, Theodoro et al. [16] and Li et al. [17] proposed the use of hybrid modeling complexes, combining the advantages of the tools for positive-sequence and electromagnetic transient modeling. This is achieved by the co-simulation of the aggregate EPS model, when the calculation is carried out in parallel in two or more DSPs, HSTs, or their combinations. Thus, it is possible to simulate large-scale EPSs with detailed models of the necessary elements: a small part of the considered EPS is simulated in detail using SP or HST for electromagnetic transient modeling, and the rest is simplified using positive-sequence modeling with all the inherent simplifications and limitations. From the presented, one of the main shortcomings naturally consists in the inability of the detailed CIG object modeling of the SIG objects, which are usually distributed over the network. At the same time, it is not possible to select the most significant of them for detailed modeling. In addition, a significant challenge regarding the distorted simulation of the mutual impact of facilities with power converters and the rest of EPS remains relevant due to its simplified modeling..

Another direction is the development of the solutions that improve the capabilities of the existing modeling tools and adapt them for the most complete and detailed simulation of CIG and EPS as a whole. In particular, a modified generic model of CIG is presented in the work of Ramasubramanian et al. [18] based on a voltage source interface. For this model, an algorithm for controlling the output current of CIG was developed, which provides a better convergence of the solution and takes account of the fast-response channels of the converter's control system. Additionally, using a WTG as an example, a model is proposed in the form of a system of ordinary differential equations in the work of Pico et al. [19], which additionally includes the main elements of the converter's control system and the dynamics of a DC-link. Yang et al. [20] proposed a new approach to describe the parts of EPS with a large share of CIG in the form of nonlinear ordinary differential equations of the fifth order, and to describe its remaining part in the form of the corresponding algebraic-differential equations. The presented solutions are aimed at using the DSPs of positive-sequence modeling. In the case of the detailed modeling of CIG and EPS as a whole using DSPs and HSTs of electromagnetic transients modeling, it is possible to identify the studies related to the development of new approaches to the numerical solution of the EPS mathematical model. For example, Wang et al. [21] used the implicit multistep high order Radau IIA method and V-transformation, which together provide high stability and speed of 
the solution. A modified diagonally implicit Runge-Kutta method of the second order is proposed for the simulation of electromagnetic transients in the work of Chakraborty et al. [22], which has significant accuracy in solving the stiff systems of differential equations that determine the EPS model.

For a comprehensive solution of the highlighted challenges of modeling modern EPSs in the context of CIG penetration, it is proposed to develop and use alternative tools that allow simulating the large-scale EPSs without their significant simplification, taking account of the detailed models of various facilities in their structure. At the same time, the modeling process itself within the framework of such tools should take account of a continuous simulation of a single spectrum of quasi-steady and transient processes in real time without decomposition. As such a tool, the hybrid real-time power system simulator (HRTSim) can be used [23]. This power system simulator is based on the concept of hybrid modeling. The features of HRTSim make it possible to exclude the decomposition of states and processes, the simplification of mathematical models of elements and the aggregate EPS model as a whole, and the limitation of the simulation time interval, as well as the truncation error of solving differential equations.

\section{A Hybrid Modeling of Electric Power System}

HRTSim is a multiprocessor multi-level modeling system. The number and composition of the simulator modules is selected in accordance with the studied EPS model. Each module is responsible for modeling a specific component of the EPS with the necessary automatic regulation and control systems. The most comprehensive and detailed three-phase mathematical models of EPS components are used, which are similar to the models for electromagnetic modeling. The detailed description of the used mathematical models is given in the work of Suvorov et al. [24]. At the same time, the implementation and calculation of these models differ from each other, which are performed in accordance with the hybrid approach. The hybrid approach combines the main existing approaches to power system modeling: analog, digital, and physical levels. The main points of the hybrid approach as abstracts are summarized in Table 1.

Table 1 Challenges and solutions

\begin{tabular}{|c|c|c|}
\hline No. & Challenges & Solutions \\
\hline 1 & $\begin{array}{l}\text { - Decomposition of the processes and regimes in EPS } \\
\text { - Simplification of the mathematical models of equipment } \\
\text { and EPS } \\
\text { - Limitation of the simulation time-intervals } \\
\text { - Variable methodological errors of numerical integration } \\
\text { of differential equations }\end{array}$ & $\begin{array}{l}\text { Analog level: } \\
\text { Application of the method of continuous } \\
\text { methodically accurate implicit integration of } \\
\text { differential equations }\end{array}$ \\
\hline 2 & $\begin{array}{l}\text { - Inadequate reproduction of switching processes } \\
\text { - Scale limitation of the modeled EPS }\end{array}$ & $\begin{array}{l}\text { Physical level: } \\
\text { Interaction of simulated EPS equipment at the } \\
\text { physical level by converting mathematical } \\
\text { variables into physical signals }\end{array}$ \\
\hline 3 & $\begin{array}{l}\text { - Operability limitation of the informational and control } \\
\text { properties and capabilities }\end{array}$ & $\begin{array}{l}\text { Digital level: } \\
\text { Application of digital-analog and analog-digital } \\
\text { conversion of information on the basis of } \\
\text { modern IT technologies }\end{array}$ \\
\hline
\end{tabular}

Such HRTSim features allow simulating the power system model of any structure and theoretically unlimited scale, which adequately take account of all significant aspects of the considered task. In particular, HRTSim allows reproducing the complex topology of the transmission and distribution networks, as well as various types and shares of CIGs with their individual characteristics. In addition, the properties and capabilities of HRTSim allow carrying out a continuous methodically accurate solution of the aggregate large-scale mathematical model of power system with CIGs with guaranteed accuracy. The accuracy of solution depends on the frequency instrumental error of the integrated microelectronic element base used in HRTSim for the analog solution. The instrumental error is $\leq 1 \%$ according to the experimental investigations of HRTSim in 
frequency range $(0-1000 \mathrm{~Hz})$ [25]. The detailed description and different performance parameters, such as the reaction time and execution time distribution of program operation via the microcontrollers used in the HRTSim, are shown in the work of Andreev et al. [23]. The reliability of the information obtained via HRTSim has been confirmed through the extensive laboratory studies and validations with field measurements [26-27]. The generic structure of HRTSim is shown in Fig. 1, where:

(1) SPs are specialized hybrid processors of various power system components, including CIGs and their control systems. The more detailed description of SPs is reported in the following section.

(2) HRTSim server is located on a main personal computer and performs the interaction with other users and SPs via the extranet and local area network (LAN), the implementation of statics and dynamics scripts, and the display of necessary simulation results and their recording.

(3) TPC refers to a three-phase commutator based on a printed circuit board, and is designed for the interconnection of three-phase inputs-outputs of SPs according to the topology of power system model, as well as for power supply of SPs.

(4) External devices are different software and hardware tools with which the information can be exchanged via the specialized hardware and software interface: supervisory control and data acquisition (SCADA) systems, economic dispatch softwares, protection devices, etc.

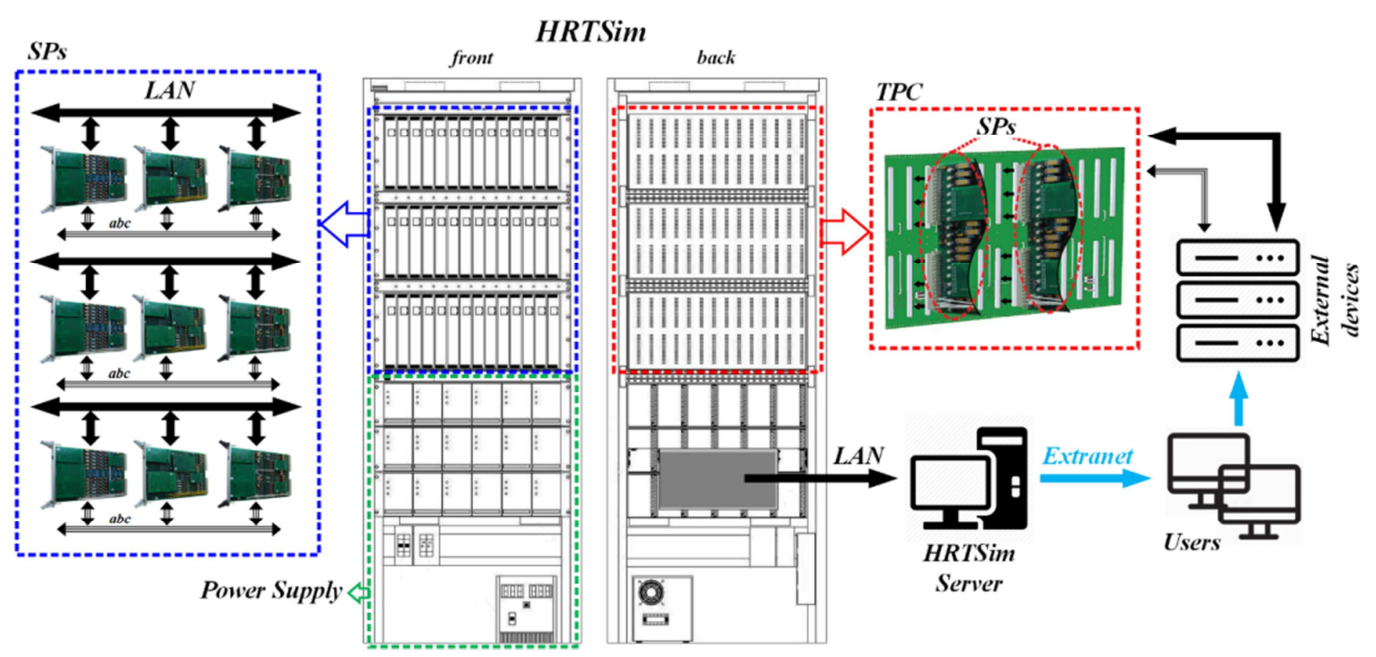

Fig. 1 Generic structure of HRTSim

\section{Implementation of Developed Hybrid Approach for CIG Simulation}

The detailed comprehensive mathematical model of CIG is developed and synthesized in the form of a SP, providing a continuous simulation of a single range of the processes in CIG (e.g., type 4 WTG in Fig. 2) as parts of the power system. The SP of PV has a similar structure, taking account of its own features. The developed SP is adapted for the use in HRTSim. Its structure and functional diagram are shown in Fig. 2, where:

\section{At the analog level:}

HCP PMG, HCP RF, HCP DC, and HCP T refer to the hybrid coprocessors (HCPs) of the mathematical models of permanent magnet generator (PMG), machine-side and grid-side reactors and low pass filters, DC-link, and step-up transformer respectively. HCPs are used for continuous and methodically accurate solution of a stiff nonlinear system of differential equations in real time with acceptable guaranteed instrumental accuracy reproducing adequate mathematical models of power system components. The results of solution are transmitted to the processor of analog-to-digital conversion (PADC). 


\section{At the digital level:}

(1) MPU refers to a microprocessor unit that provides all informational-control functions of SP of type 4 WTG and consists of several peripheral processors, the descriptions of which are provided below.

(2) CoP refers to a coprocessor for the implementation of mathematical models of wind turbine (WT), pitch angle control, machine-side and grid-side converter control, converter phase-locked loop (PLL), and protection algorithms.

(3) PDQ refers to a processor of $d q$ transformation, providing necessary non-orthogonal Park's $a b c$-to- $d q 0$ transformation and vice versa.

(4) SwP refers to a switching processor for the control of digital-controlled analog switches (DCASs) of physical models (PMs) of both series and shunt commutators (SSCs), which implements all kinds of series and shunt commutations, machine-side converters (MSCs), and grid-side converters (GSCs). SwP is also used for the pulse-width modulation process.

(5) PADC refers to a processor of analog-to-digital conversion for digitization and signal processing of simulation results via multi-channel analog-to-digital converters (ADCs).

(6) CP refers to a central processing unit for the informational and control interaction via LAN between the HRTSim server and peripheral processors, as well as the data input to the digital-to-analog converters (DACs) of HCPs and a preliminary functional processing of simulation results, if necessary.

\section{At the physical level:}

(1) VCCs refer to the integral microelectronic voltage-to-current converters of the instantaneous values of mathematical variables of input/output three-phase currents, presented as the instantaneous values of voltage in analog level, into corresponding physical currents. The interaction of physical currents relative to the simulated mathematical variables through a TPC guarantees the maximum compliance to the real power system, eliminates the problems of the digital data exchange between simulated power system components, and contributes the possibility of theoretically unlimited scale of the power system model.

(2) VFs refer to the voltage followers of the continuously generated phase voltages, which are injected into the HCPs based on microelectronic integral operational amplifiers, excluding physical impact of the feedbacks.

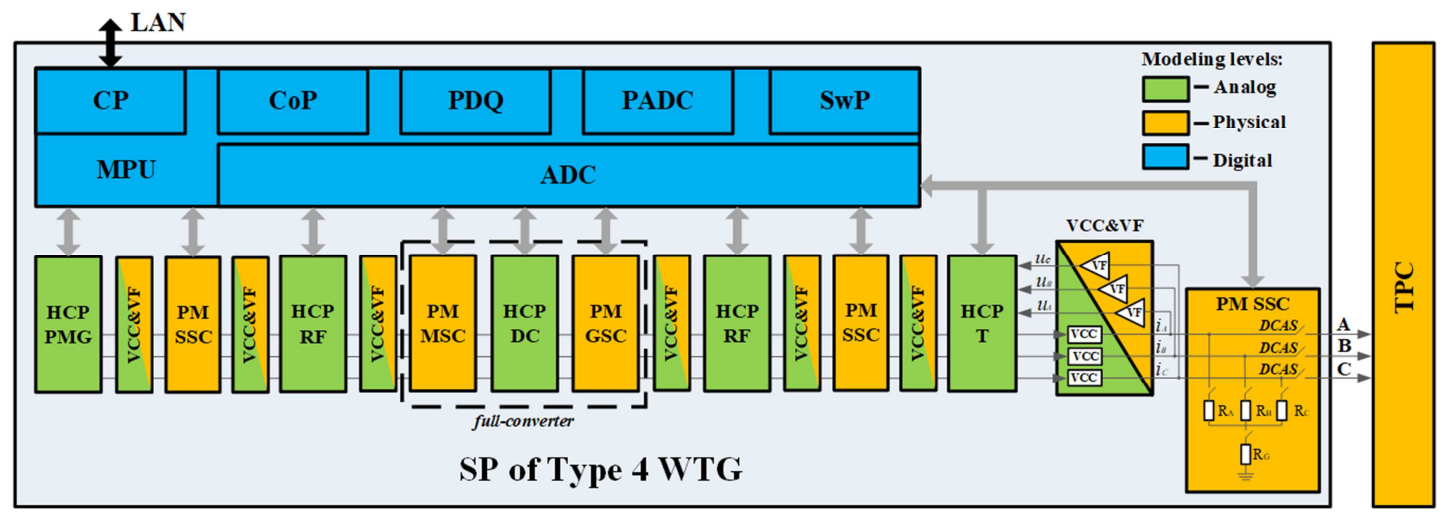

Fig. 2 Structure and functional diagram of SP of type 4 WTG

At the analog level, the formed comprehensive mathematical models of the type 4 WTG components are solved by a continuous implicit integration method, based on operational amplifier integrators, in real time and on unlimited time intervals. Since the continuous analog integration is carried out, the concept of integration step (simulation time) to the HRTSim is inapplicable, in contrast to real time digital simulators. This integration method implements a methodically accurate solution of stiff non-linear systems of differential equations. For the implementation of the above method, the specialized parallel 
digital-to-analog structures of HCPs are developed, which are the basic components of SP of type 4 WTG. The structure and functional diagram of HCP is given in Fig. 3 on the example of PMG model. Similarly, by using electronic and integral microelectronic components, other HCPs are developed and implemented.

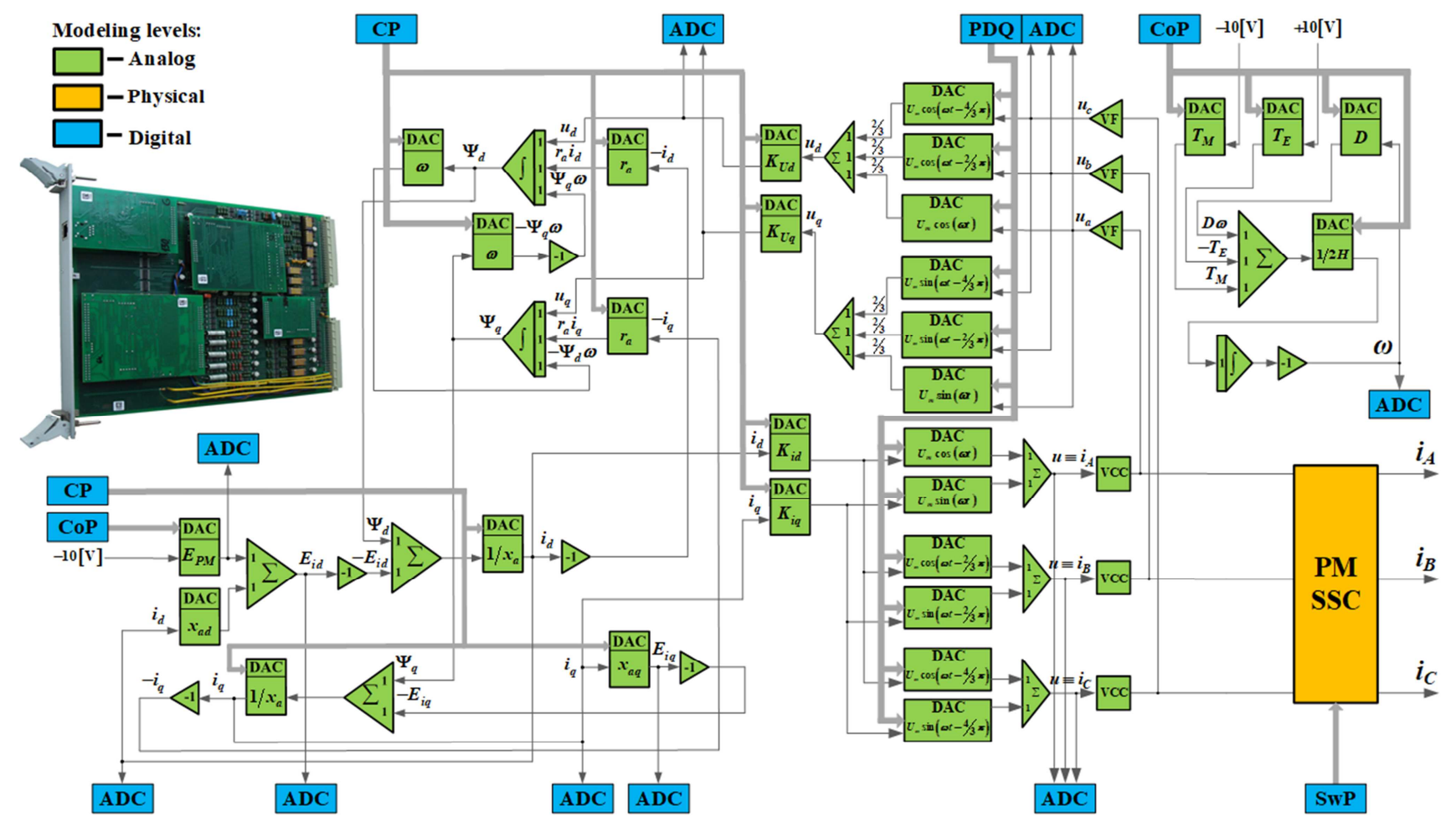

Fig. 3 Structure and functional diagram of HCP PMG

HCP PMG is developed according to Eq. (1) of the PMG model in $d q$ axes [28]. HCP RF, HCP DC, and HCP T are developed according to Eqs. (2)-(4).

$$
\left\{\begin{array}{l}
\Psi_{\delta d}=x_{a d} i_{d}, \Psi_{\delta q}=x_{a q} i_{q} ; \\
E_{i d}=x_{a d} i_{d}+E_{P M}, \quad E_{i q}=x_{a q} i_{q} ; \\
\Psi_{d}=\Psi_{\delta d}+x_{a} i_{d}, \quad \Psi_{q}=\Psi_{\delta q}+x_{a} i_{q} ; \\
i_{d}=\frac{1}{x_{a}}\left(\Psi_{d}-E_{i d}\right), i_{q}=\frac{1}{x_{a}}\left(\Psi_{q}-E_{i q}\right) \\
u_{d}=-\omega \Psi_{q}-\frac{d \Psi_{d}}{d t}-r_{a} i_{d}, u_{q}=\omega \Psi_{d}-\frac{d \Psi_{q}}{d t}-r_{a} i_{q} .
\end{array}\right.
$$

where $d \Psi_{d} / d t$ and $d \Psi_{q} / d t$ are transformation electromotive forces; $\omega \Psi_{d}$ and $\omega \Psi_{q}$ are rotational electromotive forces; $\Psi_{\delta d}$ and $\Psi_{\delta q}$ are the fluxes in the air gap; $E_{i d}$ and $E_{i q}$ are the internal electromotive forces in the air gap; $\Psi_{d}$ and $\Psi_{q}$ are the resulting fluxes; $u_{d}$ and $u_{q}$ are stator voltages; $i_{q}$ and $i_{d}$ are stator currents; $x_{a d}$ and $x_{a q}$ are unsaturated magnetizing reactances; $x_{a}$ is stator leakage reactance; $r_{a}$ is stator resistance; $E_{P M}$ is the electromotive forces of permanent magnets.

$$
\left\{\begin{array}{l}
i_{j R}=\frac{1}{R_{j}} \cdot\left(u_{j}-i_{j} \cdot R_{j C}-u_{j C}-u_{N}\right) ; \\
\frac{d i_{j L}}{d t}=\frac{1}{L_{j}} \cdot\left(i_{j R} \cdot R_{j}-i_{j L} \cdot R_{j L}\right) ; \\
i_{j}=i_{j L}+i_{j R} ; \frac{d u_{j C}}{d t}=\frac{1}{C_{j}} \cdot i_{j} ; \\
\frac{d i_{j R}}{d t}=\frac{1}{L_{j R}} \cdot\left(u_{j 1 R}-u_{j 2 R}-i_{j R} \cdot R_{j L R}\right) .
\end{array}\right.
$$


where $R_{j C}$ and $C_{j}$ are the resistance and capacitance of the capacitor bank of the $j$-th phase in the filter; $R_{j L}$ and $L_{j}$ are the resistance and inductance of the coil of the $j$-th phase in the reactor; $R_{j}$ is the resistance of the resistor of the $j$-th phase; $u_{j C}$ is the voltage on the reactance of the capacitor bank of the $j$-th phase; $u_{j 1}$ and $u_{j 2}$ are the input-output voltages of the $j$-th phase; $i_{j}$ is the current of the $j$-th phase; $R_{j L}$ and $L_{j}$ are the resistance and inductance of the $j$-th phase.

$$
\left\{\begin{array}{l}
i_{j 1 C}=\frac{1}{R_{j 1 C}} \cdot\left(u_{j 1}-u_{j 1 C}-u_{N}\right) ; \frac{d u_{j 1 C}}{d t}=\frac{1}{C_{j 1}} \cdot i_{j 1 C} ; \\
\frac{d i_{j L}}{d t}=\frac{1}{L_{j}}\left(u_{j 1}-u_{j 2}-R_{j L} \cdot i_{j L}\right) ; \\
i_{j 1}=i_{j L}+i_{j 1 C} ; i_{j 2}=i_{j \mathrm{~L}}-i_{j 2 C} ; \\
i_{j 2 C}=\frac{1}{R_{j 2 C}} \cdot\left(u_{j 2}-u_{j 2 C}-u_{N}\right), \frac{d u_{j 2 C}}{d t}=\frac{1}{C_{j 2}} \cdot i_{j 2 C} .
\end{array}\right.
$$

where $j$ is negative or positive pole; $R_{j L}$ and $L_{j}$ are the resistance and inductance of the smoothing reactor of the $j$-th pole; $R_{j l C}$ and $R_{j 2 C}$ are the resistance of the capacitor banks of the $j$-th pole; $C_{j 1}$ and $C_{j 2}$ are the capacitance of the capacitor banks of the $j$-th pole; $u_{N}$ is neutral voltage; $u_{j 1 C}$ and $u_{j 2 C}$ are the voltages on the reactance of the capacitor banks of the $j$-th pole of DC-link.

$$
\left\{\begin{array}{l}
\frac{d i_{j 1}}{d t}=\frac{1}{L_{j 1}} \cdot\left(u_{j 1}-W_{j 1} \cdot \frac{d \Phi_{j}}{d t}-R_{j 1} \cdot i_{j 1}\right) \\
\frac{d i_{j 2}}{d t}=\frac{1}{L_{j 2}} \cdot\left(W_{j 2} \cdot \frac{d \Phi_{j}}{d t}-u_{j 2}-R_{j 2} \cdot i_{j 2}\right) \\
W_{j 1} \cdot i_{\mu j}=W_{j 1} \cdot i_{j 1}-W_{j 2} \cdot i_{j 2} \\
\Phi_{j}=\frac{K_{1} \cdot i_{\mu j}}{K_{2}+K_{3} \cdot i_{\mu j}}+K_{4} \cdot i_{\mu j}
\end{array}\right.
$$

where $u_{j l}$ is the voltage of the $j$-th phase of the primary winding of the transformer; $u_{j 2}$ is the voltage of the $j$-th phase of the secondary winding, which is equal to the phase voltage in the case of star connection and to the line voltage in the case of delta connection; $i_{j 1}$ and $i_{j 2}$ are the currents of the primary and secondary windings of the $j$-th phase; $W_{j 1}$ and $W_{j 2}$ are the numbers of turns of the primary and secondary windings of the $j$-th phase; $\Phi_{j}$ is the main magnetic flux of the $j$-th phase; $i_{\mu j}$ is the magnetizing current of the $j$-th phase; $R_{j 1}$ and $L_{j l}$ are the resistances and leakage inductances of the primary windings of the $j$-th phase; $R_{j 2}$ and $L_{j 2}$ are the resistances and leakage inductances of the secondary windings of the $j$-th phase; $K_{1}, K_{2}, K_{3}$, and $K_{4}$ are the coefficients that define a particular approximated saturation curve.

At the physical level, the commutation of DCASs in PM SSC, MSC, and GSC is represented, as well as the interaction of all simulated components of power system model. The PMs of power converters are developed according to a three-level scheme of VSC [29]. The implementation of the high-speed fully controlled power semiconductor switches is carried out according to the scheme presented in Fig. 4.

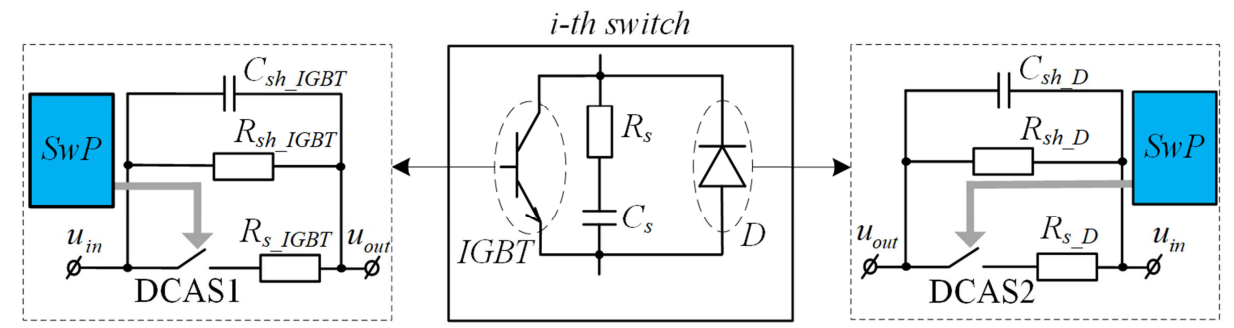

Fig. 4 Digital-controlled physical model of a high-speed fully controlled power semiconductor switch with a snubber circuit 
The unipolar DCAS1 and DCAS2 in Fig. 4 simulate the operation of the $i$-th semiconductor switch (an insulated-gate bipolar transistor (IGBT) and a bypass diode) with the corresponding parameters of their modeling circuits. Since the unipolar DCASs are almost ideal elements in the relation to the real transistor and diode, their modeling circuits are supplemented by the controlled resistances. The switches are also supplemented by the capacitances reproducing the real capacity of the $p-n$ junction of the diode and the $n-p-n$ junction of the transistor. Thus, the additional resistances and capacitances make it possible to carry out the most adequate simulation of the switching processes of the converter power switches.

At the digital level, all the informational and control functions, the automated and automatic control of parameters of the simulated power system components, and the display of the simulation results are carried out. The mathematical models of the necessary control systems and pulse-width modulation are also implemented in MPU. The mathematical model of the main elements of WT is shown in Fig. 5 [30]. In addition, all the variables of simulated components, the DCAS status, the information displaying and conversion are controlled using DACs/ADCs and specialized softwares. The necessary simulation information is processed in MPU and transmitted to the HRTSim server and users via LAN.

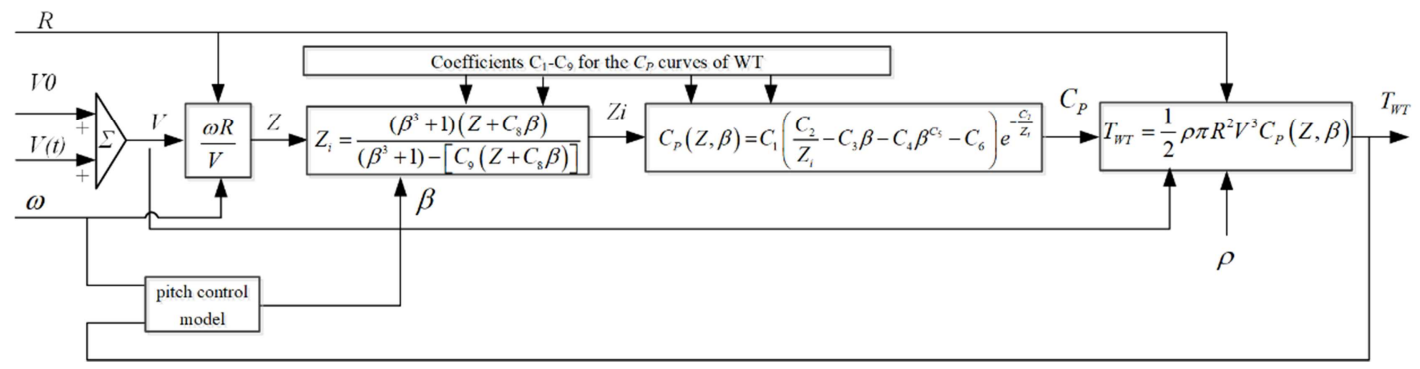

(a) Mathematical model of wind energy conversion

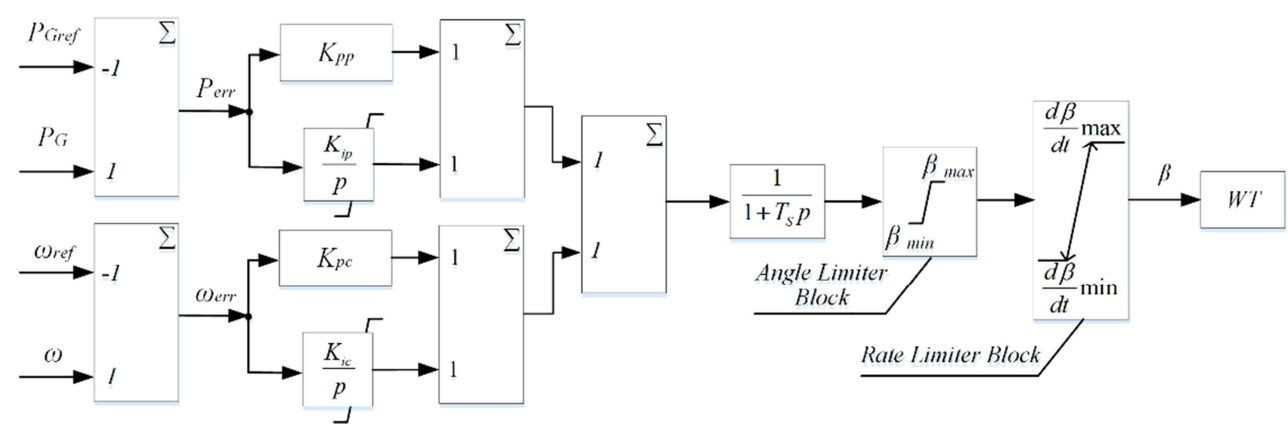

(b) Pitch control model

Fig. 5 Solved at the digital level mathematical models of the main WT elements

Note: $\rho$ is air density; $R$ is WT radius; $C_{P}(Z, \beta)$ is power coefficient; $Z$ is WT tip speed ratio; $\beta$ is blade pitch angle; $V 0$ is systematic (slowly changing) component (mean wind speed); $V(t)$ is dynamic (rapidly changing) component; $G R$ is gear ratio; $p$ is electric machine pole pairs; $T_{W T}$ is mechanical torque of WT; $K_{P P}$ and $K_{P C}$ are proportional gains of proportional integral controller; $K_{I P}$ and $K_{I C}$ are integral gains of proportional integral controller; $P_{\text {Gref }}$ and $\omega_{\text {ref }}$ are rated power and rated shaft speed of WT respectively; $\omega$ is the current shaft speed of WT; $T_{S}$ is servo motor time constant.

\section{Experimental Studies and Discussion}

The comparison of the developed SP of type 4 WTG as parts of the HRTSim with widely used and reliable tool for EPS simulation RTDS is carried out. It is important to underline that the purpose of these two simulators' comparison is to prove the adequacy of HRTSim and the developed SP, but not to show its superiority over RTDS. This validation confirms the adequacy of the developed SP because the complexity of reliable numerical integration for small test systems and the problems of large-scale EPS simulation via digital simulators are not manifested. Therefore, the results obtained via digital simulators can be considered reliable, which makes it possible to compare the HRTSim simulation results with them. The test studies are carried out in a two-machine EPS scheme, consisting of WTG, step-up transformer, power transmission line, and the load and equivalent generator with constant frequency and unlimited power. The comparisons are made for the models of all elements that form type 4 WTG and for the entire SP of type 4 WTG as a whole: 
(1) The comparative analysis of the main power characteristics of WT $\left(P_{W T}=f\left(V_{\text {wind }}\right) ; C P=f(Z)\right)$ are carried out. Comparisons are made for two cases: different pitch angles $\beta=0^{\circ}, 2^{\circ}, \ldots, 25^{\circ}$ and constant wind speed $V_{\text {wind }}=10.8 \mathrm{~m} / \mathrm{s}-C P=f(Z)$ curves (Fig. 6(a)); different pitch angles $\beta=0^{\circ}, 2^{\circ}, \ldots, 25^{\circ}$ and different wind speed $V_{\text {wind }}=0, \ldots, 25 \mathrm{~m} / \mathrm{s}-$ $P_{W T}=f\left(V_{\text {wind }}\right)$ curves (Fig. 6(b)). The values of the WT main parameters for various initial data are given in Table 2.

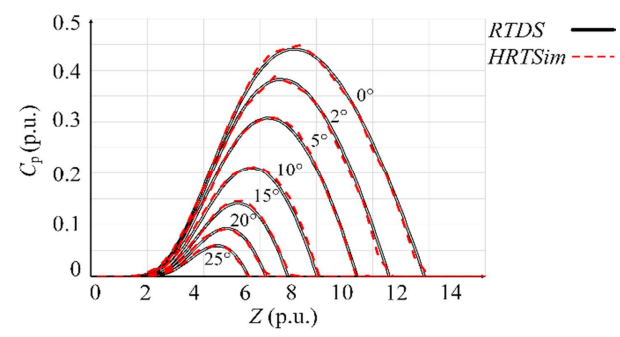

(a) $C_{P}=f(Z)$ curves for case 1

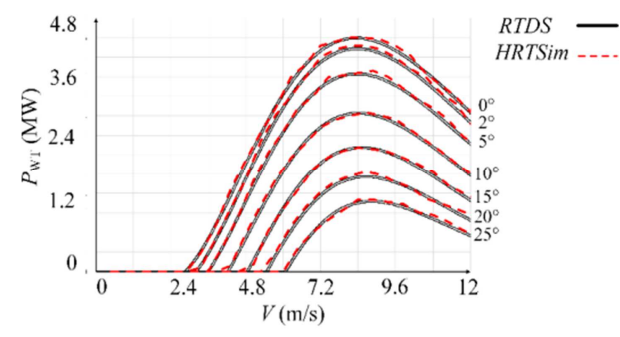

(b) $P_{W T}=f\left(V_{\text {wind }}\right)$ curves for case 2

Fig. 6 The tests of the mathematical model of the wind energy conversion of the type 4 WTG

Table 2 Comparison of SP of type 4 WTG and RTDS data (WT model tests)

\begin{tabular}{|c|c|c|c|c|c|c|}
\hline \multirow{2}{*}{ WT parameter } & \multicolumn{6}{|c|}{ Number of initial parameters } \\
\cline { 2 - 7 } & \multicolumn{4}{|c|}{1} & \multicolumn{3}{c|}{2} \\
\cline { 2 - 7 } & SP & RTDS & Error (\%) & SP & RTDS & Error (\%) \\
\hline$\omega_{W T}(\mathrm{rad} / \mathrm{s})$ & 1.375 & 1.374 & 0.073 & 1.310 & 1.309 & 0.076 \\
\hline$Z$ & 6.873 & 6.872 & 0.015 & 7.202 & 7.205 & 0.049 \\
\hline$Z_{i}$ & 6.757 & 6.733 & 0.356 & 7.068 & 7.053 & 0.213 \\
\hline$\beta(\mathrm{deg})$ & 0.0 & 0.0 & 0.000 & 0.0 & 0.0 & 0.000 \\
\hline$C_{P}$ & 0.441 & 0.438 & 0.685 & 0.439 & 0.441 & 0.499 \\
\hline$P_{W T}(\mathrm{MW})$ & 5.287 & 5.252 & 0.666 & 3.941 & 3.964 & 0.580 \\
\hline$T_{W T}$ & 0.501 & 0.500 & 0.200 & 0.393 & 0.396 & 0.758 \\
\hline
\end{tabular}

(2) In the next stage, the experimental studies of the type 4 WTG aggregate model are carried out taking account of the pitch control implemented in the SP. Fig. 7 shows the resulting pitch angle control system tests with a step change in wind speeds. In addition, the values of the WT main parameters for various wind speeds are given in Table 3.
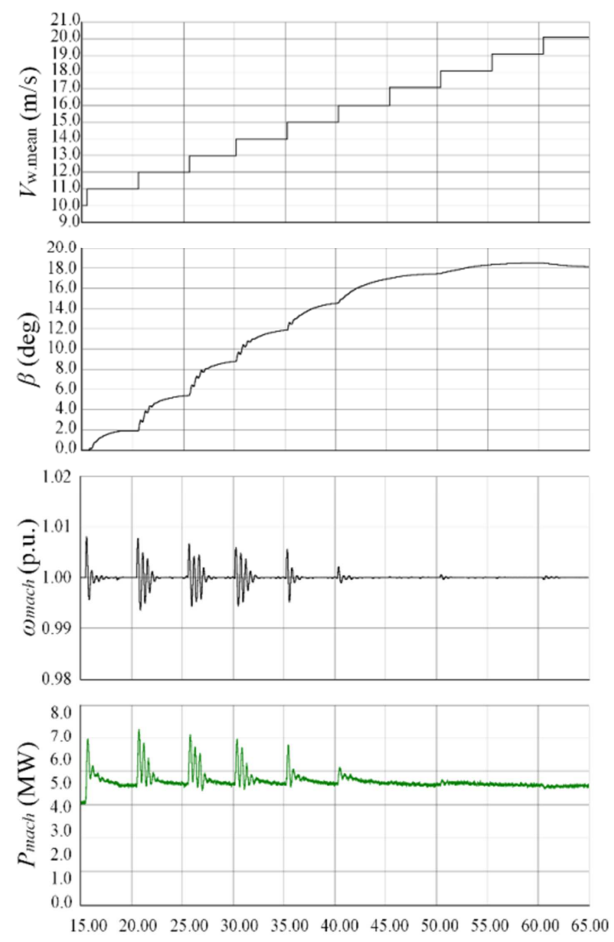

( $(\mathrm{sec})$

(a) HRTSim

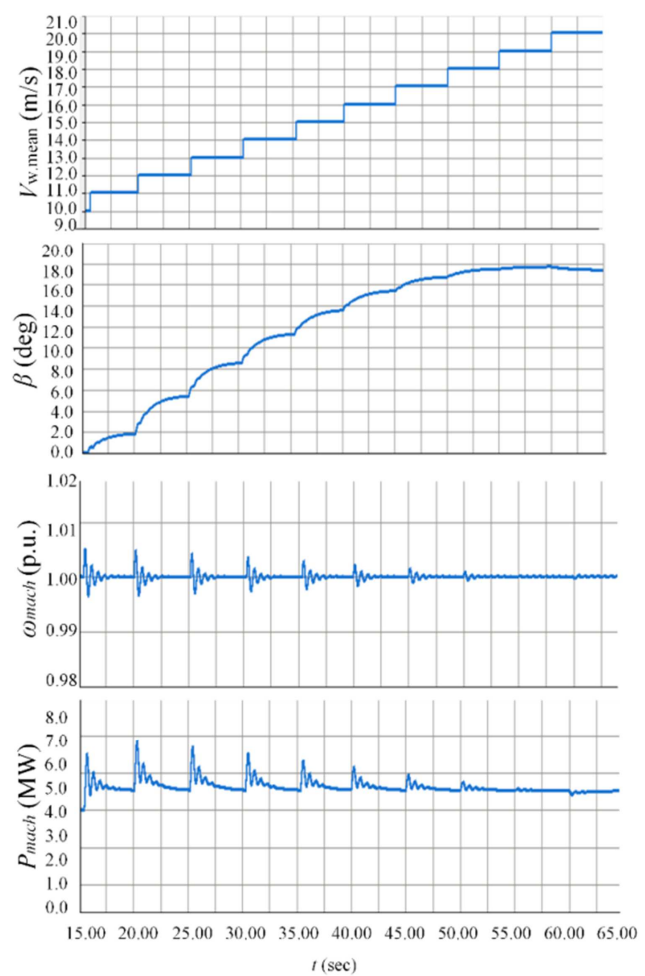

(b) RTDS

Fig. 7 Resulting oscillograms of the operation of WT pitch control in the case of steps in wind speed (from $10 \mathrm{~m} / \mathrm{s}$ up to $20 \mathrm{~m} / \mathrm{s}$ ) 
Table 3 Comparison of SP of type 4 WTG and RTDS (pitch control model tests)

\begin{tabular}{|c|c|c|c|c|c|c|c|c|c|}
\hline \multirow{2}{*}{ WT parameter } & \multicolumn{9}{|c|}{ Number of initial parameters } \\
\cline { 2 - 10 } & \multicolumn{3}{|c|}{1} & \multicolumn{3}{|c|}{2} & \multicolumn{3}{c|}{3} \\
\cline { 2 - 10 } & SP & RTDS & Error (\%) & SP & RTDS & Error (\%) & SP & RTDS & Error (\%) \\
\hline$V_{\text {wind }}(\mathrm{m} / \mathrm{s})$ & 11 & 11 & 0 & 15.8 & 15.8 & 0 & 23.3 & 23.3 & 0 \\
\hline$\beta(\mathrm{deg})$ & 1.885 & 1.855 & 1.645 & 16.065 & 15.95 & 0.721 & 14.089 & 13.994 & 0.679 \\
\hline$Z_{i}$ & 6.775 & 6.704 & 1.054 & 4.379 & 4.404 & 0.569 & 2.905 & 2.923 & 0.629 \\
\hline$Z$ & 6.763 & 6.76 & 0.048 & 4.7 & 4.706 & 0.131 & 3.186 & 3.191 & 0.166 \\
\hline$C_{P}$ & 0.388 & 0.387 & 0.351 & 0.128 & 0.13 & 1.538 & 0.041 & 0.041 & 0.784 \\
\hline$P_{W T}(\mathrm{MW})$ & 3.579 & 3.571 & 0.232 & 3.492 & 3.521 & 0.824 & 3.573 & 3.57 & 0.07 \\
\hline$T_{W T}$ & 0.715 & 0.714 & 0.12 & 0.697 & 0.704 & 0.994 & 0.715 & 0.714 & 0.126 \\
\hline$\omega_{W T}(\mathrm{rad} / \mathrm{s})$ & 1.24 & 1.239 & 0.057 & 1.24 & 1.239 & 0.081 & 1.24 & 1.239 & 0.057 \\
\hline
\end{tabular}

(3) Finally, all the elements of the type 4 WTG are tested together. The obtained waveforms in normal (Fig. 8) and emergency states (Fig. 9 and Fig. 10) are compared.

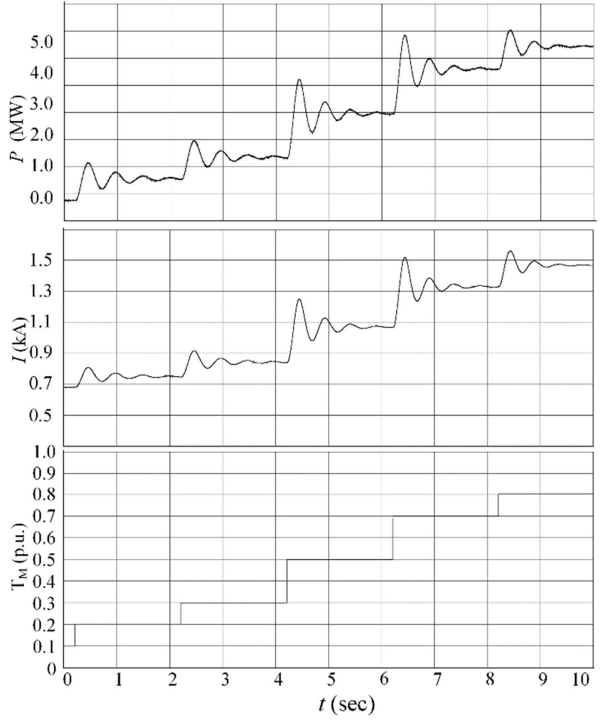

(a) HRTSim

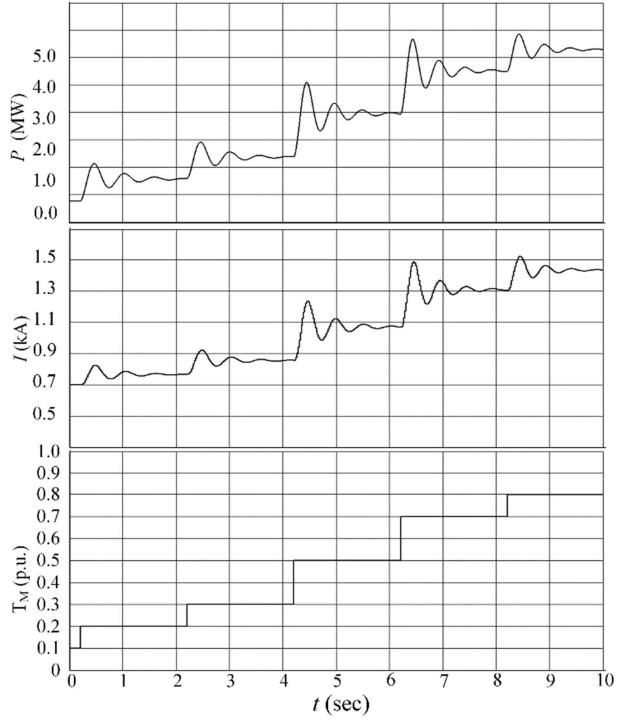

(b) RTDS

Fig. 8 Obtained waveforms during a step change of mechanical torque of WTG

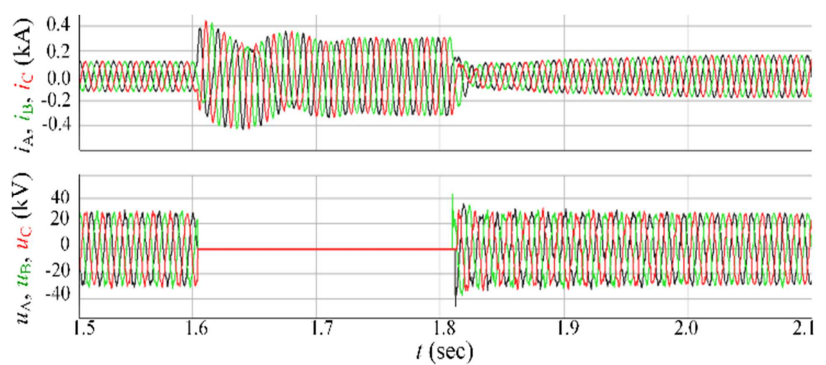

(a) HRTSim

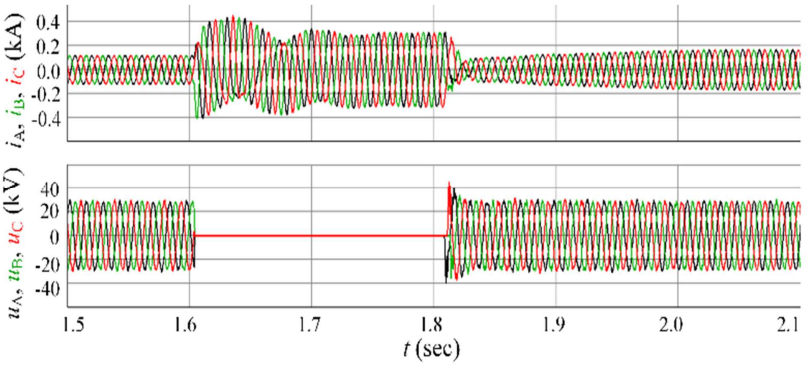

(b) RTDS

Fig. 9 Obtained waveforms during a bolted three-phase-to-ground fault at a point of common coupling of WTG

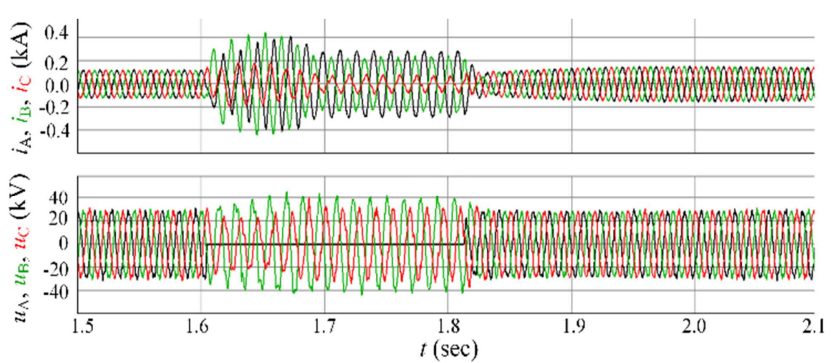

(a) HRTSim

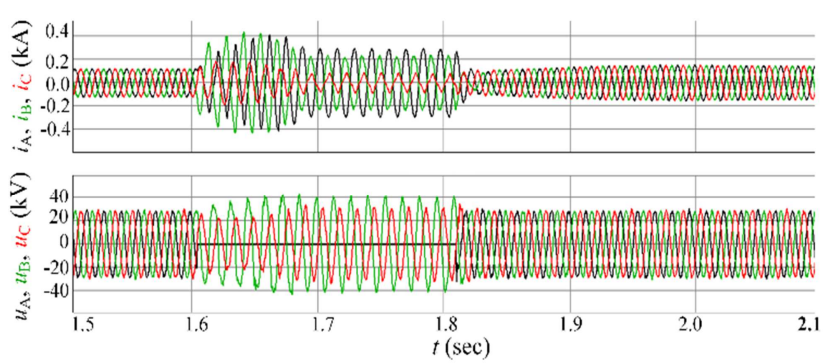

(b) RTDS

Fig. 10 Obtained waveforms during a bolted single-phase-to-ground fault at a point of common coupling of WTG 
The obtained results of all tests show the similar transient processes. The simulation error for the step change of mechanical torque is no more than $1 \%$. Also, excellent results are demonstrated by experiments with symmetrical and asymmetrical short faults. This confirms the adequacy of the developed SP and HRTSim in general.

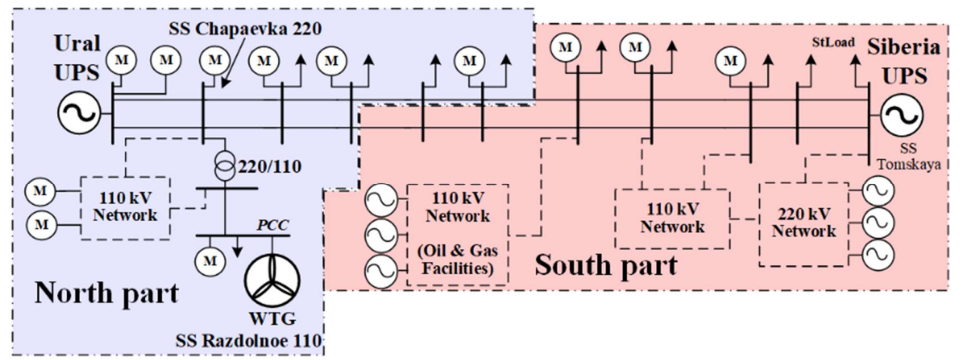

Fig. 11 Scheme of Tomsk region power system

Note: SS refers to substation; UPS refers to unified power system; StLoad refers to static load; M refers to synchronous and asynchronous motors.

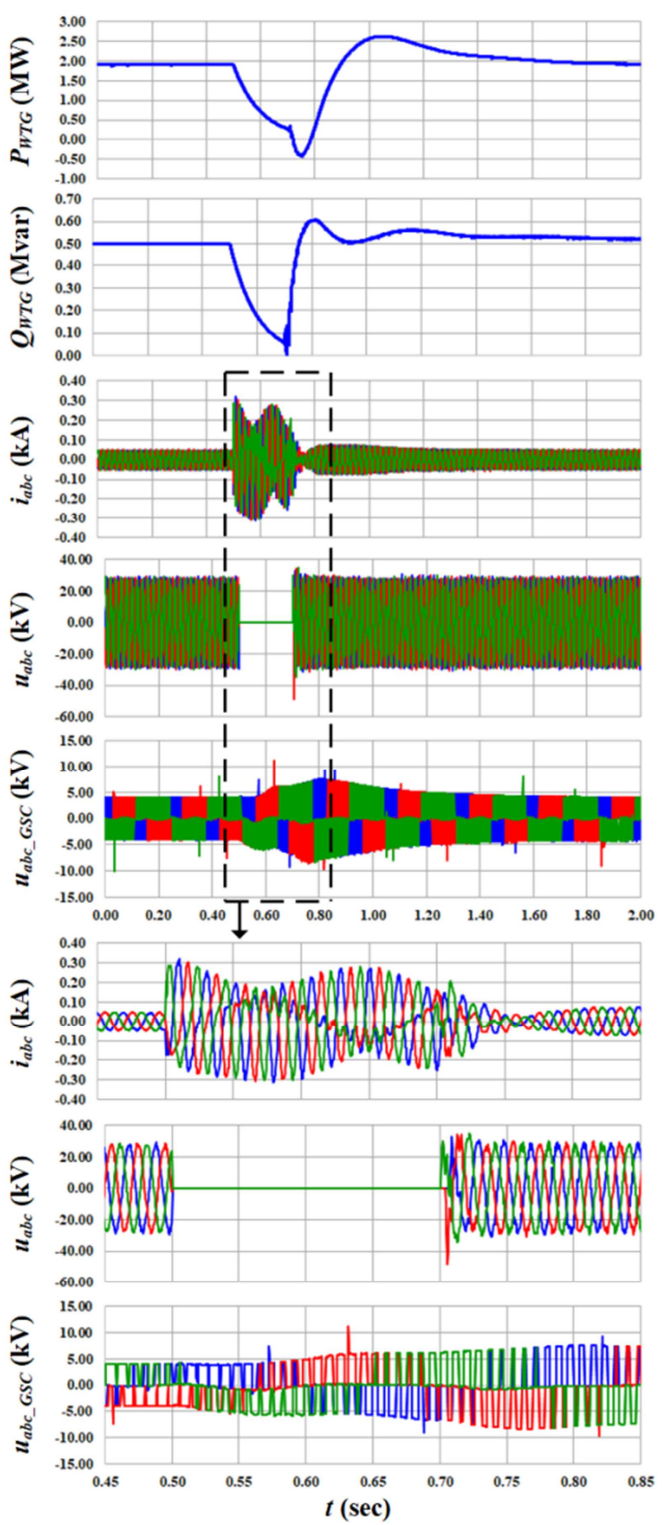

(a) Close fault

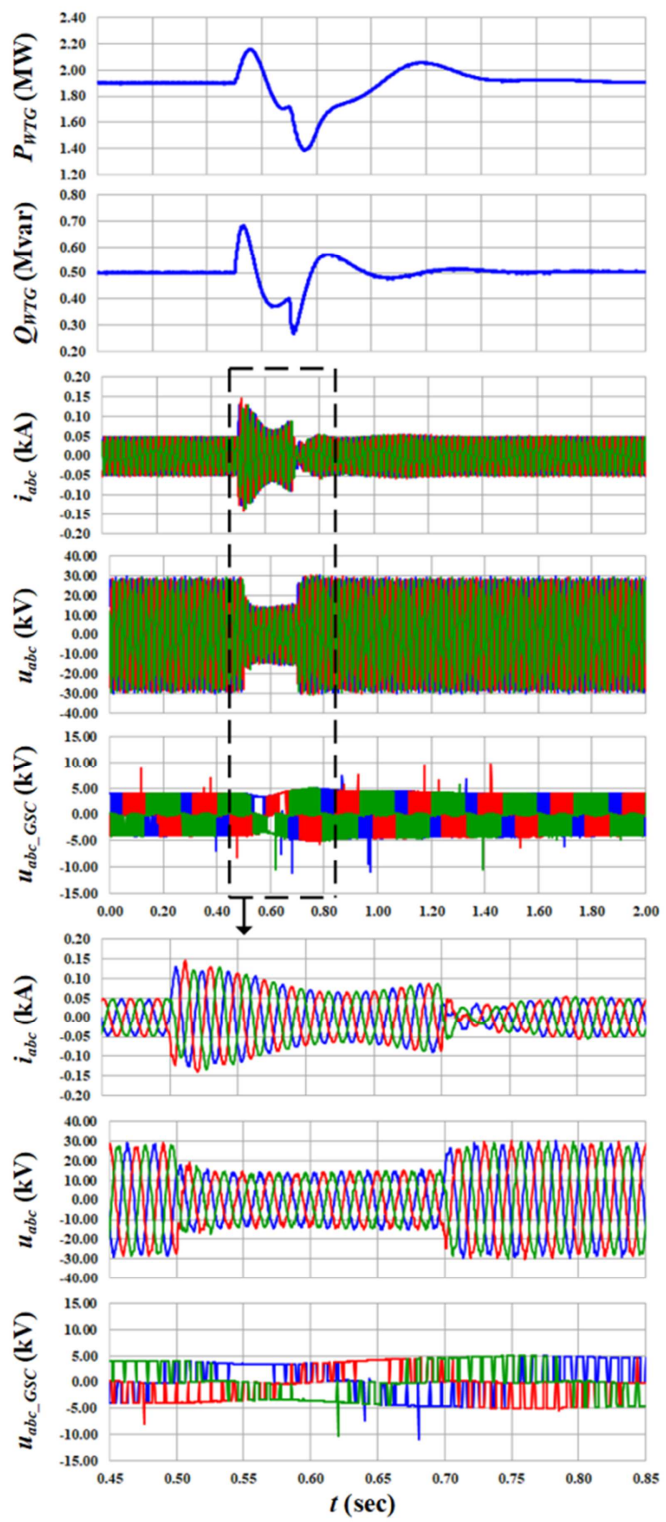

(b) Remote fault

Fig. 12 Waveforms of WTG operating parameters in the case of bolted three-phase faults

To confirm the properties, capabilities, and adequacy of the developed SP of type 4 WTG, experimental studies are carried out in the multi-machine power system model implemented in HRTSim (Fig. 11). Such testing makes it possible to assess the operation of the developed hybrid modeling tool for type 4 WTG directly as parts of the practical power system. The 
large-scale power system model in HRTSim is based on the real power system of Tomsk region, which consists of 200 three-phase nodes, 42 electric machines, 42 transformers, 97 transmission lines, and 63 static loads, including reactors and capacitor banks. The parameters of all power system components are set on the basis of the real field data and measurements. The type 4 WTG with total capacity of 2.4 MVA is installed at Substation Razdolnoe 110.

Fig. 12 shows the results of the large disturbances test: three-phase fault with a time duration of $200 \mathrm{~ms}$ at a point of common coupling (PCC) and at a remote fault near Substation Chapaevka 220 as an external disturbance test. The control system of type 4 WTG is configured to operate in $P$-priority mode with the plant level $Q$-control, whose setpoint is 0.5 Mvar. As can be seen, the obtained waveforms of dynamic response of the developed hybrid model of type 4 WTG correspond to the emerging processes, as well as to the theoretical and practical information about the transients in such WTGs in the case of disturbances. The implemented SP of type 4 WTG allows simulating modern power systems with CIGs in real time, taking account of a single continuous range of processes and a detailed large-scale model of power system. At the same time, the type, share, location, and capacity of CIGs in power system can be changed in accordance with the tasks to be solved by using appropriate SP of CIGs or their combination. In addition, the control systems of CIGs implemented at digital level are flexible that allows considering different control algorithms and structures, thereby to comprehensively analyze the behavior of CIGs in the power system, to assess different types of stability, etc.

\section{Conclusions}

The increase in the number of CIGs, in addition to positive aspects, has negative consequences associated with the operating state regulation and emergency control of modern power systems as a whole. This leads to the emergence of new challenges and problems, in particular, related to the assurance of the power system stability. The solution to these problems is possible via comprehensive studies of CIG penetration by mathematical modeling of power systems. The problems related to the studies in this field are associated with the limited capabilities of widely used simulation tools. The hybrid approach is proposed in this study, as well as the means of its implementation - HRTSim, which can simulate a single continuous range of processes in all the significant equipment and power system as a whole regardless of its scale and detail level of the simulated components. In accordance with the hybrid approach, in this study, the concept of real-time type 4 WTG modeling in the large-scale power system model is developed, and the experimental hardware-software model of SP of type 4 WTG is implemented as an example of CIG. The results of experimental studies of the developed SP are demonstrated. The reliability of the simulation results is confirmed by the coincidence with the theoretical and practical information. Thus, the use of aggregate hybrid model of power system and CIG makes it possible to analyze the features of operation of modern and promising power systems, as well as the CIG impact on operating states.

\section{Acknowledgments}

The reported study was funded by the Russian Science Foundation, project number 21-79-00129.

\section{Conflicts of Interest}

The authors declare no conflict of interest.

\section{References}

[1] Y. Cheng, R. Azizipanah-Abarghooee, S. Azizi, L. Ding, and V. Terzija, "Smart Frequency Control in Low Inertia Energy Systems Based on Frequency Response Techniques: A Review," Applied Energy, vol. 279, 115798, December 2020.

[2] A. Nouaiti, A. Saad, A. Mesbahi, and M. Khafallah, "A New Efficient Topology of Single-Phase Five-level Inverter for PV System,” International Journal of Engineering and Technology Innovation, vol. 8, no. 4, pp. 241-260, September 2018. 
[3] A. Haddadi, E. Farantatos, I. Kocar, and U. Karaagac, "Impact of Inverter Based Resources on System Protection," Energies, vol. 14, no. 4, 1050, February 2021.

[4] N. Hatziargyriou, J. V. Milanovic, C. Rahmann, V. Ajjarapu, C. Canizares, I. Erlich, et al., "Definition and Classification of Power System Stability Revisited and Extended,” IEEE Transactions on Power Systems, vol. 36, no. 4, pp. 3271-3281, July 2021.

[5] A. Suvorov, A. Gusev, M. Andreev, and A. Askarov, "A Validation Approach for Short-Circuit Currents Calculation in Large-Scale Power Systems,” International Transactions on Electrical Energy Systems, vol. 30, no. 4, e12276, April 2020.

[6] A. A. Suvorov, A. B. Askarov, M. V. Andreev, and A. S. Gusev, "Validation of Positive-Sequence Modeling of Large-Disturbance Stability in a Distribution Network with Distributed Generation Using the Hybrid Comprehensive Simulator," IEEE Access, vol. 9, pp. 65216-65233, 2021.

[7] P. Pourbeik, J. J. Sanchez-Gasca, J. Senthil, J. D. Weber, P. S. Zadehkhost, Y. Kazachkov, et al., "Generic Dynamic Models for Modeling Wind Power Plants and Other Renewable Technologies in Large-Scale Power System Studies," IEEE Transactions on Energy Conversion, vol. 32, no. 3, pp. 1108-1116, September 2017.

[8] L. Xiong, X. Liu, Y. Liu, and F. Zhuo, "Modeling and Stability Issues of Voltage-Source Converter Dominated Power Systems: A Review," CSEE Journal of Power and Energy Systems, in press.

[9] R. Agrawal, S. K. Bharradwaj, and D. P. Kothari, “An Educational and Professional Simulation Tools in Power Systems and FACTS Controllers: An Overview, “International Journal of Electrical, Electronics, and Computer Engineering, vol. 2, no. 2, pp. 91-96, 2013

[10] Y. Cai, J. Zhang, and W. Yu, “A Predictor-Corrector Method for Power System Variable Step Numerical Simulation," IEEE Transactions on Power Systems, vol. 34, no. 4, pp. 3283-3285, July 2019.

[11] Y. Lu, S. Kincic, H. Zhang, and K. Tomsovic, "Validation of Real-Time System Model in Western Interconnection," IEEE Power and Energy Society General Meeting, July 2017, pp. 1-5.

[12] X. Guillaud, M. O. Faruque, A. Teninge, A. H. Hariri, L. Vanfretti, M. Paolone, et al., "Applications of Real-Time Simulation Technologies in Power and Energy Systems," IEEE Power and Energy Technology Systems Journal, vol. 2, no. 3, pp. 103-115, September 2015.

[13] P. Pourbeik, N. Etzel, and S. Wang, "Model Validation of Large Wind Power Plants through Field Testing," IEEE Transactions on Sustainable Energy, vol. 9, no. 3, pp. 1212-1219, July 2018.

[14] C. Yang, Y. Xue, X. Zhang, Y. Zhang, and Y. Chen, "Real-Time FPGA-RTDS Co-Simulator for Power Systems," IEEE Access, vol. 6, pp. 44917-44926, 2018.

[15] J. Sun, S. Debnath, M. Saeedifard, and P. R. V. Marthi, "Real-Time Electromagnetic Transient Simulation of Multi-Terminal HVDC-AC Grids Based on GPU,” IEEE Transactions on Industrial Electronics, vol. 68, no. 8, pp. 7002-7011, August 2021.

[16] T. S. Theodoro, M. A. Tomim, P. G. Barbosa, A. C. Lima, and M. T. C. de Barros, "A Flexible Co-Simulation Framework for Penetration Studies of Power Electronics Based Renewable Sources: A New Algorithm for Phasor Extraction," International Journal of Electrical Power and Energy Systems, vol. 113, pp. 419-435, December 2019.

[17] Y. Li, D. Shu, F. Shi, Z. Yan, Y. Zhu, and N. Tai, “A Multi-Rate Co-Simulation of Combined Phasor-Domain and Time-Domain Models for Large-Scale Wind Farms,” IEEE Transactions on Energy Conversion, vol. 35, no. 1, pp. 324-335, March 2020.

[18] D. Ramasubramanian, W. Wang, P. Pourbeik, E. Farantatos, A. Gaikwad, S. Soni, et al., "Positive Sequence Voltage Source Converter Mathematical Model for Use in Low Short Circuit Systems," IET Generation, Transmission, and Distribution, vol. 14, no. 1, pp. 87-97, January 2020.

[19] H. N. V. Pico and B. B. Johnson, "Transient Stability Assessment of Multi-Machine Multi-Converter Power Systems," IEEE Transactions on Power Systems, vol. 34, no. 5, pp. 3504-3514, September 2019.

[20] Z. Yang, J. Yu, J. Kurths, and M. Zhan, "Nonlinear Modeling of Multi-Converter Systems within DC-Link Timescale," IEEE Journal on Emerging and Selected Topics in Circuits and Systems, vol. 11, no. 1, pp. 5-16, March 2021.

[21] F. Wang and M. Yang, "Fast Electromagnetic Transient Simulation for Over-Voltages of Transmission Line by High Order Radau Method and V-Transformation,” IET Generation, Transmission, and Distribution, vol. 10, no. 14, pp. 3639-3645, November 2016.

[22] S. Chakraborty and R. Ramanujam, "New Numerical Integration Methods for Simulation of Electromagnetic Transients," International Journal of Emerging Electric Power Systems, vol. 19, no. 4, 20180122, August 2018.

[23] M. V. Andreev, A. S. Gusev, N. Y. Ruban, A. A. Suvorov, R. A. Ufa, A. B. Askarov, et al., "Hybrid Real-Time Simulator of Large-Scale Power Systems,” IEEE Transactions on Power Systems,” vol. 34, no. 2, pp. 1404-1415, March 2019. 
[24] A. Suvorov, A. Gusev, N. Ruban, M. Andreev, A. Askarov, and S. Stavitsky, "The Hybrid Real-Time Dispatcher Training Simulator: Basic Approach, Software-Hardware Structure and Case Study,” International Journal of Emerging Electric Power Systems, vol. 20, no. 1, 20180165, February 2019.

[25] Y. S. Borovikov, A. S. Gusev, A. O. Sulaymanov, R. A. Ufa, A. S. Vasilev, M. V. Andreev, et al., "A Hybrid Simulation Model for VSC HVDC," IEEE Transactions on Smart Grid, vol. 7, no. 5, pp. 2242-2249, September 2016.

[26] R. Ufa, A. Gusev, A. A. Z. Diab, A. Suvorov, N. Ruban, M. Andreev, et al., "Analysis of Application of Back-to-Back HVDC System in Tomsk Electric Power System," Energy Reports, vol. 6, pp. 438-444, November 2020.

[27] M. Andreev, A. Suvorov, N. Ruban, R. Ufa, A. Gusev, A. Askarov, et al., "Settings Determination for Numerical Transformer Differential Protection via Its Detailed Mathematical Model," IET Generation, Transmission, and Distribution, vol. 14, no. 10, pp. 1962-1972, May 2020.

[28] A. B. Dehkordi, A. M. Gole, and T. L. Maguire, "Permanent Magnet Synchronous Machine Model for Real-Time Simulation," International Conference on Power Systems Transients, June 2005, pp. 1-6.

[29] R. Ufa, M. Andreev, N. Ruban, A. Suvorov, A. Gusev, I. Razzhivin, et al., "The Hybrid Model of VSC HVDC," Electrical Engineering, vol. 101, no. 1, pp. 11-18, April 2019.

[30] T. Ackermann, Wind Power in Power Systems, Chichester: John Wiley \& Sons, 2005.

Copyright $\odot$ by the authors. Licensee TAETI, Taiwan. This article is an open access article distributed under the terms and conditions of the Creative Commons Attribution (CC BY-NC) license (https://creativecommons.org/licenses/by-nc/4.0/). 